mechanism by which viral core proteins station themselves immediately on the cytoplasmic side of areas of the host cell membrane containing externally-oriented viral glycoproteins? The specificity presumably must lie in the lipid composition of these membrane patches.

Skehel reported that during infection of chick cells with fowl plague there is a build up of viral glycoproteins in the host cell plasma membrane. Insertion of large amounts of these molecules, the viral haemagglutinin and neuraminidase, into the membrane is not compensated for by a loss of host cell membrane components: all of the host cell polypeptides can be identified in apparently normal amounts in the plasma membrane fraction of virally infected cells. Again, however, as in the SV5 system the virally coded glycoproteins form discrete clusters. This was shown after sonication of plasma membrane preparations of infected cells to form vesicles. A vesicular subfraction absorbed preferentially to red blood cells and contained in addition to the expected viral haemagglutinin, the matrix protein and neuraminidase. Moreover, some host cell membrane proteins were present. By contrast, an unabsorbed subfraction contained in addition to host cell membrane proteins, the viral neuraminidase and the uncleaved haemagglutinin.

The mechanism of the cleavage process of viral envelope precursor polypeptides received considerable discussion. Perhaps the most thoroughly studied system is in cells infected with Semliki Forest virus. S. I. T. Kennedy (University of Warwick) summarised the work of the Warwick group and of other workers on the replication of Semliki Forest virus, an alphavirus, in BHK cells. When infected cells are treated with protease inhibitors (TPCK), several viral gene products accumulate intracellularly. The largest of these is a polypeptide of molecular weight 160,000 , a value that is a little short of the translational product expected of the major (26S) RNA present in virally infected cells. The polypeptide normally undergoes a series of proteolytic cleavages to produce first a polypeptide of molecular weight 120,000 and finally a core protein (molecular weight $35,000)$ and two envelope glycoproteins EP1 and EP2 (molecular weights 57,000 and 54,000). Intermediate partially cleaved polypeptides have also been identified. Pactamycin 'blocking' experiments indicate that the core polypeptide is N-terminal to the envelope polypeptides EP1.EP2. A small carbohydrate rich fragment is apparently inserted between EP1 and EP2 in the uncleaved precursor and is cut out during the fashioning by limited proteolysis.

R. Weiss (Imperial Cancer Research
Fund, London) argued for the presence of RNA tumour virus antigens on some apparently normal chick embryo cells. Cells $\left(\mathrm{GS}^{+}\right)$genetically endowed with the ability to synthesise this surface antigen, a glycoprotein support the production of infective virus (Bryan strain of RSV) whereas negative (GS-) chick cells do not. The antigen is incorporated into released virus and is necessary for subsequent attachment of virus to new host cells. Two other products studied by Weiss are coded by host genes; one of these is a surface receptor recognised by the $\mathrm{GS}^{+}$product. The other host gene specifies a surface protein product that prevents infection by blocking the receptor site when this is present. Attempts to expose the receptor in blocked cells by mild proteolysis have been unsuccessful. The inhibitor gene segregates with the $G S^{+}$ gene and it is possible that the two functions represent pleiotropic expressions of the same gene.

\section{Cytochemical approach to hormone assay}

\section{from a Correspondent}

The main conclusion of the symposium on the cytochemical approach to hormone bioassay, held at the Kennedy Institute of Rheumatology in London on December 6 , is that quantitative cytochemistry is now so precise that it can be used to measure the response produced by as little as $50 \mathrm{fg} \mathrm{ml}^{-1}$ of a hormone acting on its target cells. Consequently even $0.1 \mathrm{ml}$ of plasma is sufficient for measuring the content of corticotrophin in the blood of the patient or animal; the plasma sample is measured at two dilutions (1:100 and 1:1000) and the results should agree to $\pm 15 \%$.

Cytochemical methods for measuring the effect of four hormones are now in use. The first method, for the bioassay of corticotrophin (ACTH), is the most fully developed and the whole morning was devoted to the technique and its applications. The first part of the afternoon was concerned with the development of this method to the bioassay of luteinising hormone ( $\mathrm{LH})$ and with preliminary results concerning the levels of this hormone during the menstrual cycle. The second part of the afternoon was devoted to two of the more recent investigations-studies on the effect of thyroid stimulating hormone (TSH) and the long-acting thyroid stimulator (LATS) - and those which may lead to a new assay for gastrin.

Essentially the methods for all these assay studies are similar. The target tissue is removed from the animal and cut into segments. Each segment of tissue is maintained for $5 \mathrm{~h}$ in nonproliferative maintenance culture. This manoeuvre removes the target cells from the hormone influence of the animal and allows them to recover from the trauma of excision. The culture medium is then removed and replaced for a matter of only minutes with culture medium containing either graded concentrations of the standard hormone preparation (to obtain a response: concentration calibration graph) or one of two dilutions of the unknown plasma. The tissue is chilled, sectioned and reacted by a cytochemical method for the most appropriate biochemical activity affected by the hormone. Much of the sensitivity of these assays depends on the use of scanning and integrating microdensitometry for measuring this response solely in the target cells; moreover, they are within-animal assays so eliminating the notorious biological variability of most bioassays. The speed with which at least one of these assays can be performed has been increased remarkably by a new modification in which $20 \mu \mathrm{m}$ sections are used instead of segments of the target tissue.

The past ten years have seen a revolution in endocrinology caused by the advent of radioimmunoassay of hormones. This technique, however, has two drawbacks. In the case of certain hormones, it is very difficult to measure low physiological, or subnormal, concentrations by such methods and they sometimes detect immunopotent molecules which may have little or no biological activity. The advantages of the cytochemical bioassays are that they easily encompass the measurement of such subnormal levels and, of course, measure only biologically active molecules. In the case of ACTH the divergence of the radioimmunoassay and bioassay results became marked only during the rapid rise and fall which occurred during the hypoglycaemia stress tests, done to determine how well the hypothalamopituitary-adrenal function was maintained in rheumatoid patients treated with ACTH or with corticosteroids. A more striking divergence was seen in the levels of LH; this, if fully established, could have important implications in the present understanding of ovulation.

The techniques were first developed in the Division of Cellular Biology at the Kennedy Institute in collaboration with the Department of Chemical Pathology of the Charing Cross Hospital Medical School. They were then extended by the Department of Chemical Pathology at St Bartholomews Hospital Medical College and are currently also in use in the Department of Pharmacology, the Royal Free Hospital Medical School, and in the Department of Medicine at Newcastle. 